\title{
Espessura central da córnea: catarata congênita, pseudofacia e afacia
}

\author{
Central corneal thickness: congenital \\ cataracts, pseudophakia and aphakia
}

Weika Eulálio de Moura Santos ${ }^{1}$, José Joarez de Siqueira Junior², Gustavo Salomão ${ }^{3}$, José Ricardo Carvalho Lima Rehder ${ }^{4}$

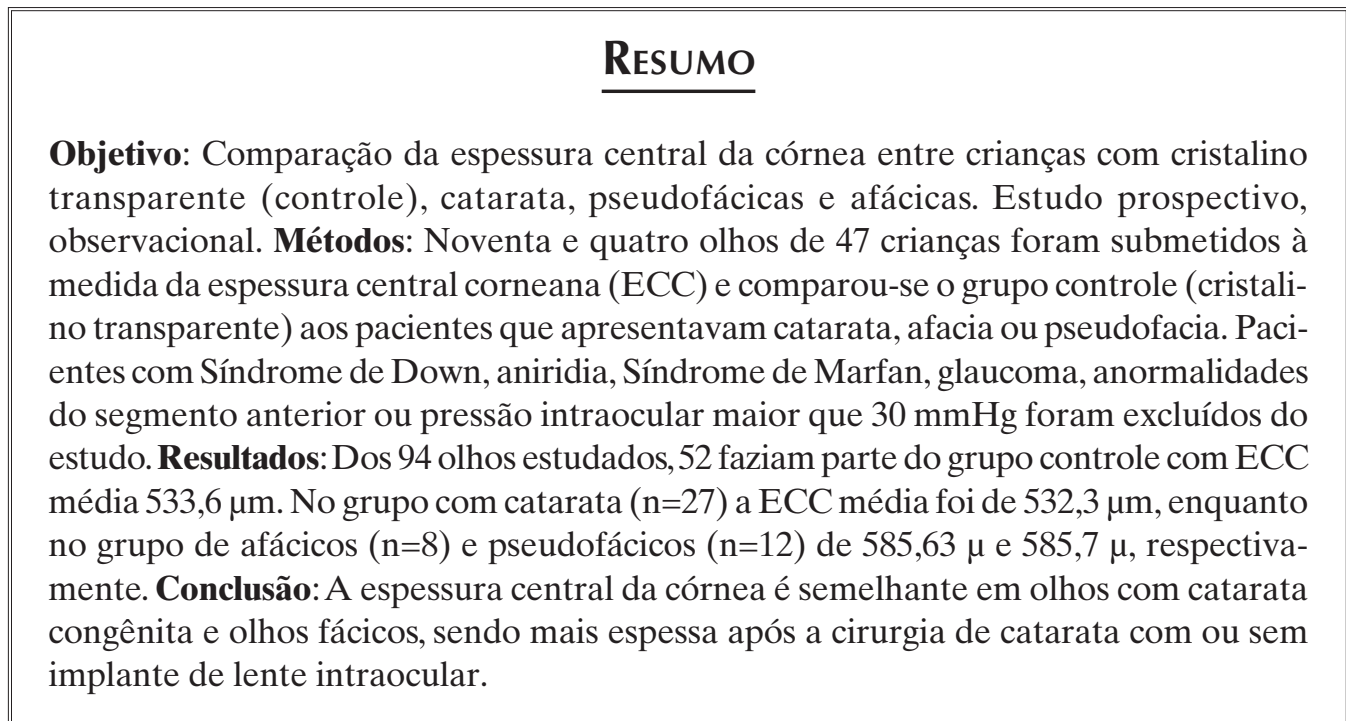

Descritores: Córnea/anatomia \& histologia; Topografia da córnea; Catarata/congênito; Pseudofacia; Afacia

\footnotetext{
${ }^{1}$ Estagiário do $3^{\circ}$ ano do Setor de Oftalmopediatria da Faculdade de Medicina do ABC - FMABC - Santo André (SP), Brasil; ${ }^{2}$ Estagiário do $3^{\circ}$ ano do Setor de Córnea e Patologia Externa da Faculdade de Medicina do ABC - FMABC - Santo Andre (SP),Brasil; ${ }^{3}$ Chefe do Setor de Oftalmopediatria da Disciplina de Oftalmologia da Faculdade de Medicina do ABC - FMABC - Santo André (SP), Brasil;

${ }^{4}$ Professor Titular da Disciplina de Oftalmologia da Faculdade de Medicina do ABC - FMABC - Santo André (SP), Brasil.

Disciplina de Oftalmologia da Faculdade de Medicina do ABC - FMABC - Santo André (SP), Brasil

Recebido para publicação em: 22/9/2009 - Aceito para publicação em 5/5/2010
} 


\section{INTRODUÇÃO}

A tonometria de aplanação de Goldmann tem sido o padrão ouro para a medida da pressão intraocular desde sua introdução em $1956^{(1)} \mathrm{e}$ pode ser falseada pela variação da espessura central corneana (ECC) em adultos ${ }^{(2,4)}$ e crianças. ${ }^{(4,6)}$

A medida da ECC através do paquímetro óptico foi descrita pela primeira vez em 1951 por Maurice e Giardini. O primeiro paquímetro ultrassônico foi descrito por Kremer em 1980. ${ }^{(7)}$

A ECC é menor na área central, aumentando em direção à periferia. A córnea da criança prematura e recém-nascida é mais espessa que a córnea do adulto; após 48 horas do nascimento, a córnea já está menos espessa e próximo aos 3 anos atinge o valor definitivo. ${ }^{(7)}$

Crianças com história de catarata congênita que são afácicas ou pseudofácicas possuem ECC maior que crianças com cristalino transparente ${ }^{(8,9)}$, mas é desconhecido se o aumento da espessura central da córnea ocorre antes da cirurgia ou se desenvolve após a mesma. O glaucoma é uma consequência comum em cirurgia de catarata congênita ${ }^{(10)}$, e uma espessura corneana fora dos limites normais talvez seja um fator importante no diagnóstico e conduta de crianças pseudofácicas e afácicas com glaucoma, pois o efeito da ECC pode falsear a medida da pressão intraocular (PIO).

\section{Métodos}

Crianças atendidas no ambulatório de Oftalmopediatria do Instituto de Olhos da Faculdade de Medicina do ABC entre agosto de 2006 a setembro 2008 participaram do estudo, sendo incluídas aquelas com história de catarata congênita, afácicas, pseudofácicas e com cristalino transparente.

Pacientes com história de cirurgia de glaucoma ou com diagnóstico conhecido de glaucoma, idade acima de 15 anos, anormalidades do segmento anterior, pressão intraocular maior que $30 \mathrm{mmHg}$, incapacidade de cooperar com a mensuração da ECC, bem como pacientes com Síndrome de Down ${ }^{(11)}$, Aniridia ${ }^{(12)}$ e Síndrome de Marfan ${ }^{(13)}$, que estão associados com uma espessura central da córnea alterada, foram excluídos do estudo.

A coleta de dados incluiu: idade, sexo, história de cirurgia, diagnóstico ocular, ECC e tonometria de aplanação de Perkins. A mensuração padrão da ECC foi realizada previamente em alguns dos pacientes do estudo. Para anestesia da córnea, cloridrato de proximetacaína $0,5 \%$ tópico foi instilado e com a sonda do paquímetro ultrassônico no centro da córnea, dez medidas foram anotadas para cada olho. O paquímetro utilizado para mensuração foi OcuScan (Alcon)®, fór- mula Herndon e a mensuração foi realizada por um único examinador.

Cada olho de cada grupo foi avaliado individualmente. Os grupos avaliados foram comparados separadamente com o grupo controle. Foi utilizado o Teste de Correlação de Spearman e o Teste de Kruskal-Wallis.

\section{Resultados}

Noventa e quatro olhos de quarenta e sete pacientes foram incluídos no estudo, sendo vinte e nove pacientes do sexo masculino e dezoito do feminino, com a média de idade de 5,5 anos (variando de 1 mês a 15 anos). Os participantes foram separados em 4 grupos: controle (Tabela 1), catarata (Tabela 2), afácicos (Tabela 3) e pseudofácicos (Tabela 4) e avaliados em relação a ECC, idade e pressão intraocular.

Dos 94 olhos, 52 faziam parte do grupo controle e a média da ECC foi 533,6 $\mu$ m. A média da ECC dos pacientes com catarata foi $532,3 \mu \mathrm{m}$, enquanto no grupo afácico e pseudofácico foi de $585,63 \mu \mathrm{m}$ e $585,7 \mu \mathrm{m}$, respectivamente (Tabela 5). No grupo controle e no grupo pseudofácico, com média de idade de 7 anos, não houve correlação significativa entre os valores de ECC, PIO e idade. Em contrapartida, nos grupos com catarata e afácicos, observamos um aumento da pressão intraocular com o aumento da idade, com média de 5 e 2,8 anos, respectivamente.

Dez olhos possuíam catarata congênita unilateral e a média da ECC do olho com catarata antes da cirurgia era maior que o olho contralateral (controle): $527,3 \mu \mathrm{m}$ e $514 \mu \mathrm{m}$, respectivamente (Tabela 6).

\section{Discussão}

No nosso estudo, os vinte pacientes submetidos à cirurgia de catarata não desenvolveram glaucoma até o presente momento. Sabe-se que olhos afácicos de crianças possuem uma espessura central da córnea maior que olhos fácicos ${ }^{(8,9)}$. Kelly et al. recentemente relataram um aumento de $45 \%$ de crianças afácicas (maior ECC) que desenvolveram glaucoma ${ }^{(14)}$. Se o aumento da ECC é um fator protetor contra o desenvolvimento do glaucoma como sugeriu Ocular Hypertension Treatment Study ${ }^{(15)}$, por que estas crianças evoluíram com glaucoma? Esses autores relataram também que um maior tempo de afacia se correlacionou com uma maior média na espessura central da córnea ${ }^{(14)}$. Nilforushan e associados relataram em um estudo semelhante, um aumento na ECC em crianças afácicas comparadas com crianças fácicas de idade e contagem celular endotelial semelhante. ${ }^{(16)}$ Porém, não observamos no presente estudo correlação entre duração do estado de afacia e au- 
Tabela 1

\section{Grupo controle}

\begin{tabular}{llr}
\hline & Descritiva & \\
\hline ECC & Média & 533,6 \\
& Desvio padrão & 44,7 \\
& Mínimo & 449 \\
PIO & Máximo & 632 \\
& Média & 11,7 \\
& Desvio padrão & 2,2 \\
Idade & Mínimo & 6 \\
& Máximo & 16 \\
& Média & 7,3 \\
& Desvio padrão & 3,5 \\
& Mínimo & 9 \\
& Máximo & 15,0 \\
\hline
\end{tabular}

Nesse grupo $(\mathrm{n}=52)$ não houve correlação significante entre os valores de ECC, PIO e Idade;

Teste de Correlação de Spearman ECC x PIO: $\mathrm{r}=-0,143 ; \mathrm{p}=$ 0,312 / ECC x Idade: $r=0,065 ; p=0,646 /$ PIO x Idade: $r=0,232$; $\mathrm{p}=0,098$

Tabela 3

\section{Grupo afácico}

\begin{tabular}{llr}
\hline & Descritiva & \\
\hline ECC & Média & 585,63 \\
& Desvio padrão & 54,764 \\
& Mínimo & 510 \\
PIO & Máximo & 662 \\
& Média & 10,50 \\
& Desvio padrão & 2,726 \\
Mdade & Máximo & 7 \\
& Média & 13 \\
& Desvio padrão & 2,875 \\
& Mínimo & 3,6354 \\
& Máximo & 1 \\
& & 10,0 \\
\hline
\end{tabular}

Nesse grupo $(\mathrm{n}=8)$ houve correlação significante entre os valores de PIO e Idade; Teste de Correlação de Spearman ECC x PIO: $\mathrm{r}=$ $-0,128 ; p=0,763$ / ECC x Idade: $r=-0,024 ; p=0,955 /$ PIO x Idade: $\mathrm{r}=0,910 ; \mathrm{p}=0,002$ (aumento de PIO com aumento de idade)

mento da ECC durante um ano de acompanhamento.

Verificamos que olhos pseudofácicos $(585,7 \mu \mathrm{m}[\mathrm{n}=$ 12]) e olhos afácicos $(585,63 \mu \mathrm{m}[\mathrm{n}=8])$ possuíam maior ECC que olhos fácicos $(533,6 \mu \mathrm{m}[\mathrm{n}=52])$ e olhos com catarata $(532,3 \mu \mathrm{m}[\mathrm{n}=27])$.

A influência da idade na ECC não é clara. Day e Gunderson $^{(17)}$ não encontraram diferença na ECC entre um grupo de crianças mais jovens com 2 anos de idade e um grupo com 18 anos de idade. Neste estudo, no grupo controle e no grupo pseudofácico (média de idade de 7 anos) não houve correlação significativa entre os valores de ECC, PIO e idade. Entretanto, nos grupos com catarata e afácicos, observamos um aumento da pressão intraocular com o aumento da idade (5 e 2,8 anos res-
Tabela 2

Grupo com catarata

\begin{tabular}{llr}
\hline & Descritiva & \\
\hline ECC & Média & 532,3 \\
& Desvio padrão & 45,0 \\
& Mínimo & 449 \\
PIO & Máximo & 621 \\
& Média & 10,7 \\
& Desvio padrão & 2,8 \\
Idade & Mínimo & 7 \\
& Máximo & 15 \\
& Média & 5 \\
& Desvio padrão & 3,9 \\
& Mínimo & 1 \\
& Máximo & 15,0
\end{tabular}

Nesse grupo $(\mathrm{n}=27)$ houve correlação significante entre os valores de PIO e Idade; ECC x PIO: $r=0,071 ; p=0,726$ / ECC $x$ Idade: $\mathrm{r}=-0,213 ; \mathrm{p}=0,287 /$ PIO $\mathrm{x}$ Idade: $\mathrm{r}=0,533 ; \mathrm{p}=0,004$ (aumento de PIO com aumento de idade)

\section{Tabela 4}

\section{Grupo pseudofácico}

\begin{tabular}{llr}
\hline & Descritiva & \\
\hline \multirow{2}{*}{ ECC } & Média & 585,7 \\
& Desvio padrão & 79,6 \\
& Mínimo & 487 \\
PIO & Máximo & 730 \\
& Média & 11,4 \\
& Desvio padrão & 2,7 \\
Idade & Mínimo & 7 \\
& Máximo & 14 \\
& Média & 7,0 \\
& Desvio padrão & 2,8 \\
& Mínimo & 3,0 \\
& Máximo & 10,0 \\
\hline
\end{tabular}

Nesse grupo ( $n=12)$ não houve correlação significante entre os valores de ECC, PIO e Idade; Teste de Correlação de Spearman ECC $\times$ PIO: $r=-0,157 ; p=0,627 /$ ECC $\times$ Idade: $r=0,403 ; p=$ 0,194 / PIO x Idade: $r=0,556 ; p=0,060$

pectivamente). Nesses dois grupos (catarata e afácicos) a média de idade era menor, sendo necessário avaliação sob sedação inalatória em alguns casos, podendo ter ocorrido influência do anestésico na aferição da PIO.

A diferença na ECC entre os grupos sugere que o aumento da espessura central da córnea ocorre após cirurgia. Porém, a razão deste aumento da ECC ser devido aos danos causados às células endoteliais durante a remoção da catarata ou ser consequência do estado de afacia ou pseudofacia permanece desconhecido. Uma avaliação, a longo prazo, mais detalhada da ECC antes e após a extração da catarata seria necessária para elucidar esta questão; e assim avaliar seu papel no desenvolvimento de glaucoma nessas crianças. 
Tabela 5

Teste de Kruskal-Wallis referente à espessura central da córnea (ECC)

\begin{tabular}{lcr}
\hline Grupo & N & Média ECC \\
\hline Controle & 52 & 533,58 \\
Catarata & 27 & 532,33 \\
Cirurgia sem lente & 8 & 585,63 \\
Cirurgia com lente & 12 & 585,67 \\
\hline
\end{tabular}

Significante $(\mathrm{X} 2=9,266 ; \mathrm{p}=0,026)$; Houve diferenças significativas entre os valores médios de ECC, quando comparamos os grupos entre si. Os grupos submetidos à cirurgia, com ou sem lente, apresentam valores médios de ECC maiores que os dos grupos controle e com catarata

Tabela 6

Teste de Correlação de Spearman

\begin{tabular}{lcr}
\hline & Descritiva & \\
\hline $\mathbf{N}$ & Controle & Catarata \\
\hline $\mathrm{N}$ & 10 & 10 \\
Média & 514,0 & 527,3 \\
\hline
\end{tabular}

Significante $(r=0,685 ; p=0,029)$; Houve correlação significante e positiva entre os valores de ECC, quando comparamos o olho com catarata antes da cirurgia e o olho controle

\section{Abstract}

Purpose: To evaluate central corneal thickness (CCT) in phakic children (controls) and in those with cataracts, pseudophakia and aphakia.Study prospective, observational Methods: Central corneal thickness was measured in 94 eyes of 47 children. Subjects with Down Syndrome, aniridia, Marfan Syndrome, glaucoma, anterior segment abnormalities or intraocular pressure over $30 \mathrm{mmHg}$ were excluded. Groups were compared for controls and for eyes with pediatric cataracts, pseudophakia and aphakia. Results: Twenty-nine patients were male and eighteen female and the mean age was 5.5 years (range, 1 month to 15 years). Of the 94 eyes, 52 were part of the control group and the CCT average was $533.6 \mu \mathrm{m}$. The average measurement of the $C C T$ for all patients with cataract was $532.3 \mu \mathrm{m}(n=27)$. The average CCT aphakia the group was $585.63 \mu \mathrm{m}(n=8)$ and pseudophakic was $585.7 \mu m(n=12)$. Conclusion: In the absence of factors known to affect CCT (Down syndrome, aniridia, and Marfan syndrome), CCT is similar in eyes with pediatric cataracts and normal controls and increases after cataracts surgery.

Keywords: Cornea/anatomy \& histology; Corneal topography; Cataract/congenital; Pseudophakia; Aphakia

\section{REFERÊNCIAS}

1. Goldmann H. Applanation tonometry. In: Newell F, editor. Glaucoma: transactions of the second conference. New York: Josiah Macy, Foundation; 1956. p.167-220.

2. Ehlers N, Hansen FK, Aasved H. Biometric correlations of corneal thickness. Acta Ophthalmol (Copenh).1975;53(4):652-9.

3. Herndon LW, Choudhri SA, Cox T, Damji KF, Shields MB, Allingham RR. Central corneal thickness in normal, glaucomatous, and ocular hypertensive eyes. Arch Ophthalmol. 1997;115(9):1137-41. Comment in: Arch Ophthalmol. 1998;116(4):544-5; Arch Ophthalmol. 1998;116(5):701; Arch Ophthalmol. 1998;116(9):1263.

4. Muir KW, Jin J, Freedman SF. Central corneal thickness and its relationship to intraocular pressure in children. Ophthalmology. 2004;111(12): 2220-3.

5. Hussein MA, Paysse EA, Bell NP, Coats DK, Brady McCreery $\mathrm{KM}$, et al. Corneal thickness in children. Am J Ophthalmol. 2004; 138(5):744-8.

6. Belfort Jr R, Kara-José N. Córnea: clínica cirúrgica. São Paulo:Rocca; 1997.

7. Yamane R. Semiologia ocular. Rio de Janeiro: Cultura Médica; 2003. Curvatura, paquimetria e microscopia especular da córnea. p. 89-102.

8. Simon JW, O'Malley MR, Gandham SB, Ghaiy R, ZobalRatner J, Simmons ST. Central corneal thickness and glaucoma in aphakic and pseudophakic children. J AAPOS. 2005;9(4):326-9.

9. Simsek T, Mutluay AH, Elgin U, Gursel R, Batman A. Glaucoma and increased central thickness in aphakic and pseudophakic patients after congenital cataract surgery. Br J Ophthalmol. 2006;90(9):1103- 6.

10. Keech RV, Tongue AC, Scott WE. Complications after surgery for congenital and infantile cataracts. Am J Ophthalmol. 1989;108(2):136-41.

11. Evereklioglu C, Yilmaz K, Bekir NA. Decreased central corneal thickness in children with Down syndrome. J Pediatr Ophthalmol Strabismus. 2002;39(5):274-7.

12. Brandt JD, Casuso LA, Budenz DL. Markedly increased central thickness: an unrecognized finding in congenital aniridia. Am J Ophthalmol. 2004;137(2):348-50.

13. Sultan G, Baudouin C, Auzerie O, De Saint Jean M, Goldschild M, Pisella PJ. Cornea in Marfan disease: Orbscan and in vivo confocal microscopy analysis. Invest Ophthalmol Vis Sci. 2002;43(6):1757-64.

14. Kelly W. Muir, Lois D, Laura B, David K, Sharon F. Central corneal thickness: congenital cataracts and aphakia. Am J Ophthalmol. 2007;144(4):502- 6.

15. Brandt JD, Beiser JA, Kass MA, Gordon MO. Central corneal thickness in the Ocular Hypertension Treatment Study (OHTS). Ophthalmolgy. 2001;108(10):1779-88.

16. Nilforushan N, Falavarjani KG, Razeghinejad MR, Bakhtiari P. Cataract surgery for congenital cataract: endothelial cell characteristics, corneal thickness, and impact on intraocular pressure. J AAPOS. 2007;11(2):159-61.

17. Dai E, Gunderson CA. Pediatric central corneal thickness variation among major ethnic populations. J AAPOS. 2006;10(1):22-5.

Endereço para correspondência:

Weika Eulalio de Moura Santos

Rua Dr. Eduardo Amaro, no 99, Apto 1814 - Paraíso

CEP 04104-080 - São Paulo (SP),Brasil

Tel: (11) 8343-3843

E-mail: weika80@hotmail.com 